\title{
Künstliche Intelligenz im Marketing: Möglichkeiten und Herausforderungen
}
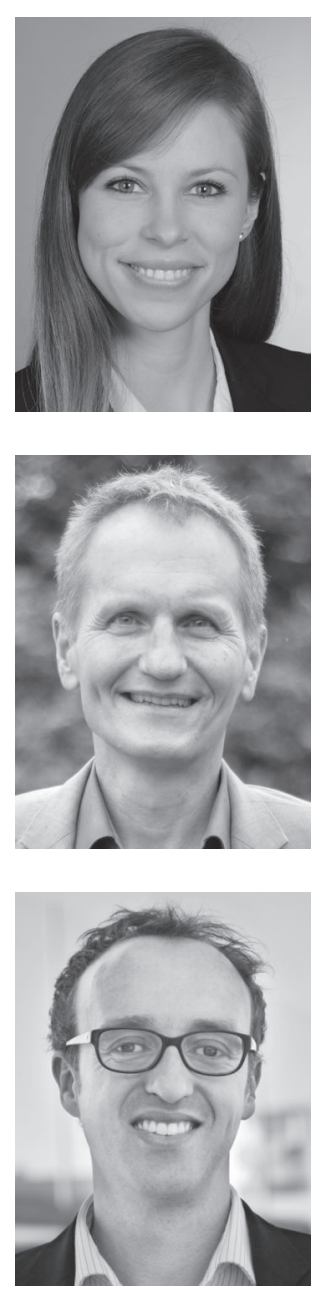

\section{Gioia Volkmar, Sven Reinecke und Peter Mathias Fischer}

Zusammenfassung: Künstliche Intelligenz (KI) gewinnt an Bedeutung bei der Lösung vieler Aufgaben, die früher von Menschen ausgeführt wurden. So planen viele Unternehmen in naher Zukunft KI-Anwendungen einzuführen, vernachlässigen aber dabei die ganzheitliche Implementierung in die Unternehmensstrategie.

Im Rahmen der internationalen, qualitativ ausgerichteten Trendstudie "Künstliche Intelligenz im Marketing" wurden Möglichkeiten und Herausforderungen der KI in ihrer Anwendung im Marketing untersucht. $\mathrm{Zu}$ diesem Zweck wurden 39 Interviews mit Experten aus den Bereichen Wissenschaft, Technologie und Management durchgeführt. Zum einen wurden Möglichkeiten und Grenzen sowie Herausforderungen bezüglich der Anwendung von KI im Marketing unterschiedlicher Branchen ausgelotet. Zum anderen wurden Faktoren diskutiert, die das Management in ihrer Erwartungshaltung, ihren Entscheidungen und der Implementierung von KI-Technologien beeinflussen. Nachfolgend werden die zentralen Ergebnisse der Studie hinsichtlich der Herausforderungen und Möglichkeiten der KI im Marketing aufgezeigt.

Stichwörter: Künstliche Intelligenz, Marketing, Management, KIImplementierung, KI-Trends, KI-Herausforderungen, KI-Möglichkeiten, KI-Ethik

\section{Artificial Intelligence in Marketing: Opportunities and Challenges}

Summary: Artificial Intelligence (AI) is becoming increasingly important in solving many tasks that were previously performed by humans. Many companies are planning to introduce AI applications in the near future, but tend to neglect the holistic implementation in their corporate strategy.

As part of an international, qualitative trend study on "Artificial Intelligence in Marketing", the possibilities and challenges of AI in its application in marketing were examined. For this purpose, 39 interviews with experts from academia, technology, and management were carried out. On the one hand, possibilities and limits as well as challenges relating to the application of $\mathrm{AI}$ in marketing in various industries were explored. On the other hand, factors were discussed that influence management in terms of expectations, decisions, and implementation of AI technologies. In the following, the 
main results of the study regarding the challenges and possibilities of AI in marketing are revealed.

Keywords: Artificial Intelligence, Marketing, Management, AI Implementation, AI Trends, AI Challenges, AI Opportunities, AI Ethics

\section{KI als multidisziplinäres Thema}

In Zukunft wird künstliche Intelligenz (KI) zu starken Veränderungen in vielen Lebensbereichen führen (Wolan 2020). KI hat bereits mehrere Branchen vorangebracht (Torra et al. 2019). Ihr Einsatz umfasst sowohl verschiedene Verbraucheranwendungen als auch verschiedene industrielle Anwendungen, einschliesslich Gesundheitswesen, Handel, Finanzdienstleistungen und Marketing (Huang et al. 2018).

Die KI-Revolution wird durch den Einfluss von immer grösseren Datenmengen sowie einer steigenden Rechenleistung zur Verarbeitung von Daten in Echtzeit vorangetrieben (Gentsch 2019). KI-Anwendungen ermöglichen besonders im Marketing Erkenntnisse aus den umfassenden Informationen zu gewinnen. Diese Informationen sind auf unterschiedlichen Kanälen (Web, Mobil oder persönlich) verteilt und umfassen verschiedene Formen (Kommentaren oder Blogs) (Kietzmann et al. 2018). Mit Hilfe dieser Informationen kann KI genutzt werden, um zum Beispiel personalisierte Empfehlungen (in Bezug auf Produkte oder den optimalen Preis) in Echtzeit zu liefern (Davenport et al. 2020). Dadurch können Unternehmen ihren Service verbessern und personalisiert auf die individuellen Kundenbedürfnisse eingehen (Rust 2019). Der Einsatz verschiedener KI-Anwendungen ermöglicht es Marketingführungskräften, neue und zuverlässigere Informationen zur Prozessautomatisierung, Marktprognose und Entscheidungsfindung zu nutzen (Colson 2019).

Um ein bestmögliches Ergebnis zu erzielen, sollten Mensch und KI allerdings zusammenarbeiten. Damit stellt sich die Frage, wie eine solche Zusammenarbeit aussehen könnte (Traumer et al. 2017). Ein kritischer Aspekt besteht darin, dass Menschen häufig das grundlegende Verständnis fehlt, was KI bedeutet und welche Potentiale diese Technologie mit sich bringt (Davenport/Kirby 2016).

\section{Künstliche Intelligenz verstehen}

Trotz einer langen Geschichte der KI, die ihre Anfänge bereits 1956 auf der Dartmouth Summer Conference hatte, gibt es bis heute keine universelle Begriffsdefinition (Russel/ Norvig 2019). Zahlreiche Forscher definieren KI nach den Kriterien, ob ein System wie ein Mensch denkt (Bellman 1978), wie ein Mensch reagiert (Kurzweil 1990; Rich/Knight 1991) oder ob Systeme rational denken (Charniak/McDermott 1985) beziehungsweise rational agieren (Nilsson 1998). Es lassen sich aber auch Gemeinsamkeiten in den Definitionen finden:

(1) Technologischer Fokus: Ob Computer, Maschinen, Algorithmen oder Roboter: Alle sind in der Lage, zu denken, zu handeln, die Umgebung wahrzunehmen und daher komplexe Aufgaben selbständig zu lösen (McCartby et al. 1955; Nilsson 1998; Kaplan/Haenlein 2019).

(2) Menschlicher Fokus: Es wird eine gewisse Intelligenz von technischen Systemen verlangt, um eine Aufgabe auszuführen, wie es auch von einem Menschen erwartet wird (Kurzweil 1990; Chui et al. 2018; Wilson/Daugherty 2018). 
$\mathrm{Da}$ es sich bei der KI um ein komplexes Thema handelt und sich die Technologie dynamisch entwickelt, hat sich noch keine einheitliche Definition durchgesetzt. Eine Möglichkeit besteht darin, drei Schlüsselaspekte ins Zentrum zu stellen:

(1) Verstehen ist die menschliche Wahrnehmung und Interpretation von Umweltinformationen, beispielsweise mittels natürlicher Sprachverarbeitung und Computer Vision (Chui et al. 2018; Russel/Norvig 2019).

(2) Schlussfolgern lässt sich als Modell interpretieren, das eine bestimmte Eintrittswahrscheinlichkeit angibt. Dies wird genutzt, um fundierte Entscheidungen zu treffen oder Empfehlungen abzugeben (Bellman 1978; Kolbjørnsrud et al. 2017).

(3) Lernen bedeutet, dass die KI die Fähigkeit besitzt, aus verschiedenen Arten von Informationen Rückschlüsse zu ziehen und sich an die jeweilige Umgebung anzupassen, was intelligentes Verhalten aufzeigt (McCarthy et al. 1955; Kurzweil 1990).

Diese drei Aspekte kombinieren unterschiedliche Fähigkeiten von KI, die den Menschen hinsichtlich seines Denkens und Handelns unterstützen sollen. Mangels Verfügbarkeit einer allgemeingültigen Definition des Begriffs KI, definieren wir diese anhand der drei Schlüsselaspekte:

"Künstliche Intelligenz als Zweig der Wissenschaft und Technik ist in der Lage, unterschiedliche Aufgaben intelligent zu erfüllen, Fehler zu erkennen und aus diesen zu lernen. Somit besitzt KI die Fähigkeit, in einem unsicheren Umfeld intelligentes Verhalten anzunehmen und angemessen zu handeln. "

Diese Definition zeigt, dass KI eine multidisziplinäre Wissenschaft ist.

\section{Methodische Vorgehensweise im Rahmen der KI-Trendstudie}

\subsection{Datenerhebung}

Zielsetzung: Das Ziel der Studie bestand darin, aktuelle und zukünftige Trends sowie Herausforderungen der Anwendung von KI im Marketing zu identifizieren. Aus diesem Grund wurde eine Trendstudie mit 39 ausgewählten Experten aus Wissenschaft, Technologie und Management durchgeführt, die fundierte Kenntnisse über KI-Fähigkeiten und -Anwendungen haben. Die internationale qualitative Studie fokussierte schwerpunktmässig, aber nicht ausschliesslich, auf den deutschsprachigen Raum.

Selektion der Experten: Gemäss Liebold und Trinczek (2009) verfügt ein Experte über spezifische Kenntnisse in einem abgegrenzten Wissensgebiet. Somit erfolgte die Auswahl der Experten in drei Schritten. Im ersten Schritt wurden selektiv Experten anhand vorher festgelegter Kriterien ausgewählt:

(1) Geschäftsgebiete: Die Interviewpartner sollten relevante Branchen repräsentieren. Darunter fallen Technologiekonzerne, Unternehmensberatungen, Wissenschaft und Forschung.

(2) Technologie: Zu jedem KI-Technologiebereich gemäss der Technologiegrafik (siehe Abbildung 1) sollten mindestens zwei Experten befragt werden.

(3) Marketing Management: Experten sollten Manager aus dem Bereich Marketing sein, welche Kenntnisse über KI besitzen. 
Diese Kriterien waren wichtig, um das Forschungsfeld zu strukturieren und anschliessend einen umfassenden Einblick zu KI im Marketing zu erhalten (Wassermannn 2015). Im zweiten Schritt wurde das Schneeballverfahren angewandt. Die Experten wurden gebeten, weitere Interviewpartner zu benennen, welche denselben Kriterien entsprechen (Palinkas et al. 2015). Im dritten Schritt wurden solange Interviews geführt, bis eine Saturation der Erkenntnisse eintrat, welche gemäss Morse et al. (2002) ein Indikator für die Reliabilität der Daten darstellt.

Insgesamt lag die Antwortrate im europäischen Raum bei $77 \%$ und im asiatischen Raum bei 13 \%. Die Rückmeldequote der Grundlagenwissenschaftler war dabei erwartungsgemäss geringer als jene der anwendungsorientierten Wissenschaftler. Um eine mögliche Verzerrung zu vermeiden, wurde beim Ausfall eines Experten ein anderer Interviewpartner explizit aus demselben Bereich rekrutiert. Insgesamt wurden Experten aus den folgenden Bereichen interviewt: Wissenschaft und Forschung (6 Teilnehmende), Marketing (9), Technologie (14) und Unternehmensberatungen (10). Die Experten wurden auch zu Trends und Beispielen in Bezug auf KI befragt, die für sie aus subjektiver Perspektive interessant waren. Sie galten nicht als stellvertretend für das Unternehmen, sondern lediglich als Experten für eine KI-Technologie (vgl. Abbildung 1). Es wurden keine expliziten persönlichen Fragen gestellt, um die Offenheit und Flexibilität der Experten nicht zu gefährden.

Datenerhebung: Die Daten wurden mittels Telefon- und Skype-Interviews erhoben. Die Interviews folgten einem semi-strukturierten Leitfaden, um die neuen Ideen und Themen innerhalb der Gesprächsdauer (30-90 Minuten) zu ermöglichen (Jaeger/Reinecke 2009). Es kamen drei Interviewer zum Einsatz, die über verschiedenes Hintergrundwissen zu KI verfügen (Betriebswirtschaftslehre, Psychologie und Ingenieurwesen/Technologie). Unser Ziel war es, dadurch den Einfluss von Suggestivbefragungen und/oder einen Interviewer Bias zu minimieren. Zudem wurde anhand einer Literaturanalyse sichergestellt, dass alle Interviewer über eine ausreichende inhaltliche Kompetenz verfügten. Die Interviews begannen mit Fragen zu Trends, Chancen und Herausforderungen der KI im Marketing. Der zweite Teil konzentrierte sich auf Faktoren, die den Entscheidungsprozess von Führungskräften beeinflussen, einschliesslich ethischer Aspekte.

Methode: Alle Interviews wurden mit Zustimmung der Teilnehmenden aufgezeichnet und transkribiert. Aufgrund des explorativen Charakters wurde der Begriff KI nicht vorgängig spezifiziert und für die Interpretation durch die Teilnehmenden bewusst offengehalten. Vorläufige Themenbereiche wurden basierend auf den gewonnenen Daten und den von den Teilnehmenden gelieferten Informationen generiert. Der Schwerpunkt jedes Interviews wurde durch die Antworten der einzelnen Experten bestimmt und angepasst. Zusätzlich wurde eine Grafik (vgl. Abbildung 1) der relevantesten KI-Technologien als Orientierungshilfe für die Experten zur Verfügung gestellt.

\subsection{KI-Technologiegrafik}

Wie bereits im Kapitel 2 diskutiert, gibt es zahlreiche Definitionen der KI, die sowohl Unterschiede als auch Gemeinsamkeiten aufweisen. Aus diesem Grund und angesichts des explorativen Charakters der Studie wurde den Teilnehmenden vor dem Interview bewusst keine Definition vorgegeben. Dadurch blieben die Experten unvoreingenommen und konnten ihre eigene Definition von KI äussern und begründen. 
Auf der Grundlage einer disziplinenübergreifenden Literaturrecherche wurde eine Technologiegrafik (vgl. vereinfacht Abbildung 1) entwickelt und den Experten als Orientierungshilfe bereitgestellt. ${ }^{1}$ Auf dieser Basis wurden die Interviewfragen von den Teilnehmenden beantwortet. Der Grounded Theory (Glaser/Strauss 1967) folgend wurde ein iteratives Vorgehen gewählt, bei welchem die Grafik basierend auf dem Expertenfeedback kontinuierlich angepasst und validiert wurde.

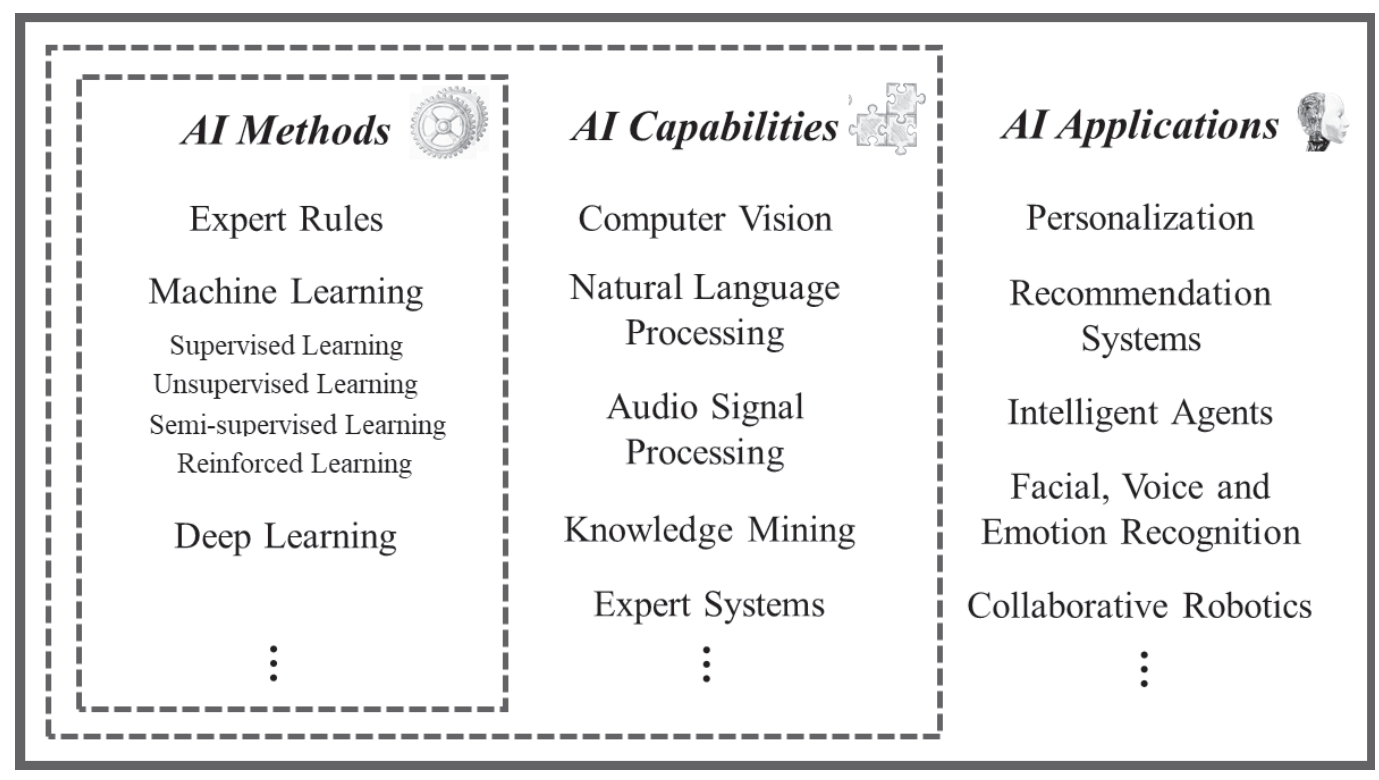

Abbildung 1: KI-Technologiegrafik ${ }^{2}$

Abbildung 1 illustriert einen Ausschnitt relevanter KI-Technologien. ${ }^{3}$ Diese lassen sich in drei Bereiche gliedern: 1) KI-Methoden (AI Methods), 2) KI-Fähigkeiten (AI Capabilities) und 3) KI-Anwendungen (AI Applications).

KI-Methoden dienen dazu, unterschiedliche Arten von Daten zu verarbeiten und zu strukturieren. Dabei stellen Machine Learning und Deep Learning Teilbereiche der KI dar. Sie verwenden statistische Methoden, um Systeme mit der Fähigkeit zum Lernen auszustatten. (Torra et al. 2019). Abbildung 2 zeigt verschiedene Machine Learning-Methoden.

1 Die Technologiegrafik wurde in englischer Sprache allen Experten vorgelegt.

2 Eigene Darstellung basierend auf Russel/Norvig 2009; Daugherty/Wilson 2018; Gentsch 2019.

3 Die aufgelisteten Aspekte repräsentieren einen Teil von KI-Methoden, KI-Fähigkeiten und KI-Anwendungen, die heute verwendet werden. 


\begin{tabular}{|c|c|c|c|}
\hline & Description & Type of Data & Method \\
\hline $\begin{array}{l}\text { Supervised } \\
\text { Learning }\end{array}$ & $\begin{array}{l}\text { It learns by following } \\
\text { a predetermined target } \\
\text { pattern }\end{array}$ & Labeled data & $\begin{array}{l}\text { Maps the labeled input } \\
\text { to the known output }\end{array}$ \\
\hline $\begin{array}{l}\text { Unsupervised } \\
\text { Learning }\end{array}$ & $\begin{array}{c}\text { The systems acts on } \\
\text { unsorted input data } \\
\text { without guidance }\end{array}$ & Unlabeled data & $\begin{array}{l}\text { Understands patterns } \\
\text { and discovers the } \\
\text { unknown output }\end{array}$ \\
\hline $\begin{array}{l}\text { Semi-supervised } \\
\text { Learning }\end{array}$ & $\begin{array}{l}\text { It learns from labeled } \\
\text { data while classifying } \\
\text { unlabeled data }\end{array}$ & $\begin{array}{l}\text { Combination of labeled } \\
\text { data and unlabeled data }\end{array}$ & $\begin{array}{l}\text { Use of existing, } \\
\text { assigned data to label } \\
\text { unlabeled data }\end{array}$ \\
\hline $\begin{array}{l}\text { Reinforced } \\
\text { Learning }\end{array}$ & $\begin{array}{l}\text { It learns based on the } \\
\text { feedback from its } \\
\text { environment }\end{array}$ & No predefined data & $\begin{array}{l}\text { Follows the trial and } \\
\text { error method }\end{array}$ \\
\hline
\end{tabular}

Abbildung 2: Unterteilung der Machine Learning-Methoden ${ }^{4}$

KI-Fähigkeiten basieren auf KI-Methoden und ermöglichen Systemen, die Umwelt zu verstehen. Bekannte Beispiele für KI-Fähigkeiten sind unter anderem Computer Vision oder Natural Language Processing. Mittels Computer Vision kann die Umgebung visuell erkannt werden. Dies ermöglicht Objekterkennung und Bildanalyse durch visuelle Daten aus der Umgebung. Natural Language Processing ermöglicht es der KI, die natürliche Sprache zu verstehen (Gentsch 2019).

KI-Anwendungen basieren auf KI-Methoden und/oder KI-Fähigkeiten und beschreiben Applikationen in der Praxis. Diese Anwendungen können dann vom Endnutzer genutzt werden, seien es Führungskräfte, Mitarbeitende oder Kunden. Darunter fallen zum Beispiel Intelligente Agenten, Personalisierung und Empfehlungssysteme. Intelligente Agenten (Intelligent Agents) interagieren mit dem Menschen und verstehen Anfragen mit Hilfe von Natural Language Processing. Bekannte Beispiele sind Alexa oder Siri (Gentsch 2019). Personalisierung (Personalization) ordnet Merkmale einem Nutzer zu und passt im Anschluss Programme, Dienste oder Informationen an die persönlichen Vorlieben, Bedürfnisse und Fähigkeiten des Nutzers an (Daugherty/Wilson 2018). Empfehlungssysteme (Recommendation Systems) schlagen dem Kunden neue Produkte vor. Die empfohlenen Produkte basieren dabei auf vergangenen Interaktionen mit dem System und ermöglichen eine zielgerichtete Empfehlung. Bekannte Anwendungen sind Netflix oder Spotify (Gentsch 2019).

Der bereits in Abbildung 1 eingeführte Zusammenhang zwischen KI-Methoden, KI-Fähigkeiten und KI-Anwendungen ist jedoch nicht trennscharf zu unterscheiden, sondern ist abhängig von den unterschiedlichen Einsatzfeldern. Grundsätzlich bilden die KI-Fähigkeiten den Transmissionsriemen zwischen KI-Methoden und KI-Anwendungen (Russel/ Norvig 2009; Daugherty/Wilson 2018; Gentsch 2019).

4 Eigene Darstellung basierend auf Russel/Norvig 2009; Daugherty/Wilson 2018; Torra et al. 2019. 


\subsection{Kodierung der Experteninterviews}

Die Transkripte, die zusammengefassten Erkenntnisse aus den Interviews sowie die zur Verfügung gestellten Informationen wurden analysiert und nach der von Gioia et al. (2013) vorgeschlagenen Vorgehensmethodik kodiert. Basis für das Kodierungsschema waren die Interviewfragen sowie ausgewählte Aussagen der Experten. Dieses Schema ermöglichte eine offene Kodierung und diente als Bezugsrahmen, um eine systematische Auswertung der Ergebnisse sicherzustellen.

Die Aussagen der Experten wurden im ersten Schritt als Konzepte erster Ordnung zusammengefasst. Auf diese Weise liessen sich im Anschluss die Themen zweiter Ordnung identifizieren und entsprechend klassifizieren (zum Beispiel: "Efficiency" oder "Acceptance"). Diese Kategorien wurden dann zu übergeordneten Dimensionen "Chances" und "Challenges" aggregiert. In diesem Artikel werden wir den Fokus auf die Möglichkeiten sowie die Herausforderungen der KI legen (vgl. Abbildung 3).
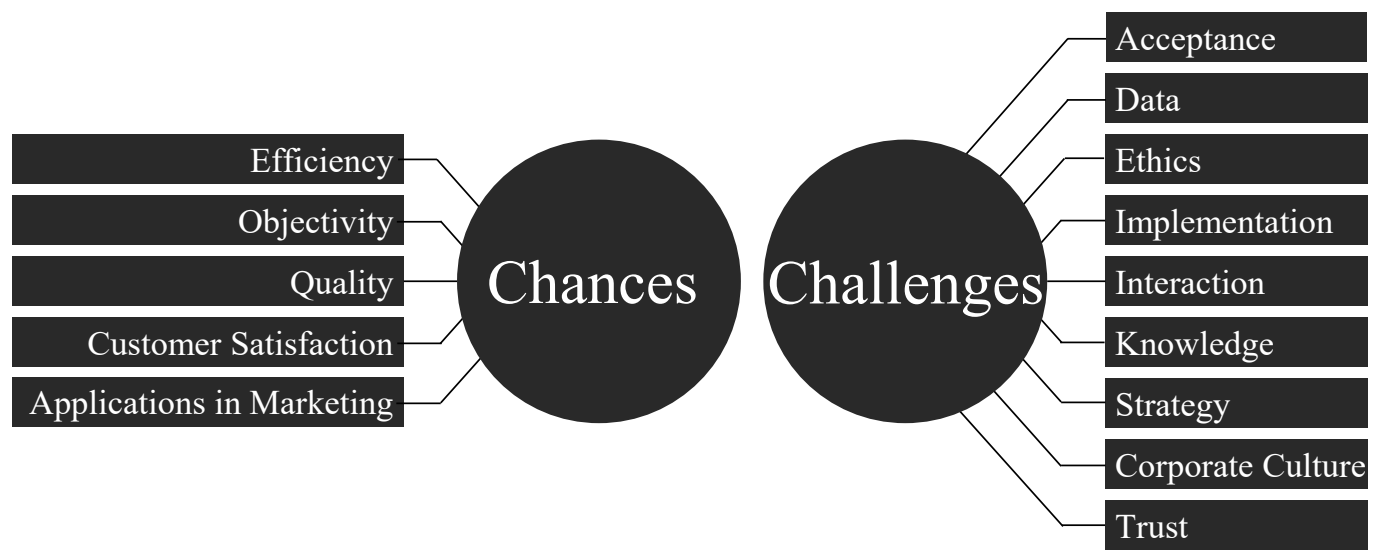

Abbildung 3: Kodierungsschema der Möglichkeiten und Herausforderungen der $\mathrm{KI}^{5}$

Nach Gioia et al. (2013) erfolgte im nächsten Schritt die Untersuchung der daraus entstehenden Themen und Konzepte. Dabei diente die Kodierung der Ergebnisse als Basis für die Identifizierung der als relevant eingeschätzten Themenschwerpunkte. Im Zuge der Typenbildung erfolgte eine fokussierte, systematische Betrachtung einzelner Aspekte. Es wurden anhand dieser Gruppierung fünf Kategorien entwickelt. Im nächsten Kapitel wird detailliert auf die identifizierten Themenfelder der KI eingegangen.

\section{Zentrale Ergebnisse der KI-Trendstudie}

\subsection{Themenfelder der KI im Marketing}

Auf Grundlage der Ergebnisse der Experteninterviews (vgl. Kapitel 3.3) wurden fünf Themenfelder der KI identifiziert (vgl. Abbildung 4).

5 Die Auswertung sowie die Kodierung erfolgten auf Englisch. 


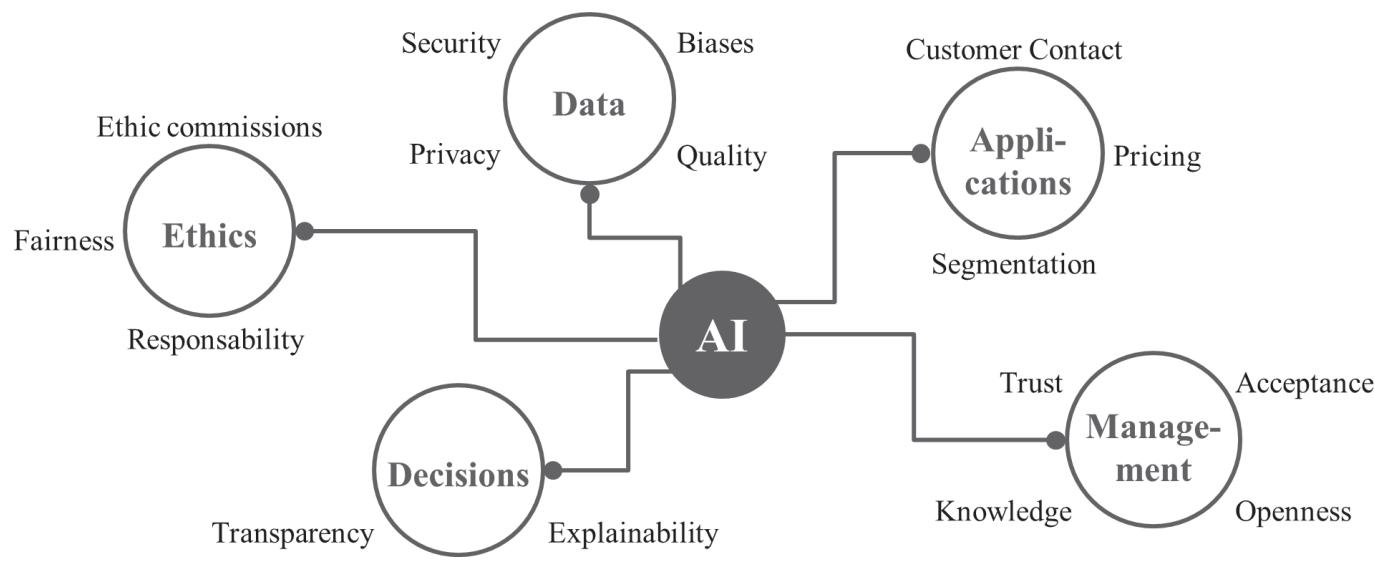

Abbildung 4: Identifizierte Themenfelder der KI

\section{Daten}

Bezüglich der Datenbasis werden Herausforderungen der KI sowohl bei der Datenerhebung als auch bei der -verarbeitung gesehen. Eine mögliche Gefahr liegt in einer zu geringen Quantität und Qualität der Daten. Dadurch besteht die Möglichkeit, dass die KI Falsches lernt und somit verzerrte Ergebnisse produziert. Ein Beispiel hierfür ist das Unternehmen Amazon, das mithilfe von KI den Bewerbungsprozess effizienter gestalten wollte. $\mathrm{Da}$ die KI mit den Lebensläufen der derzeitigen und mehrheitlich männlichen Angestellten geschult wurde, waren die Daten verzerrt (biased data), so dass die KI Bewerbungen von Frauen überwiegend ablehnte.

Des Weiteren sind Datenschutz und Privatsphäre der Nutzer grosse Herausforderungen, welche auch einen Bezug zu ethischen Fragen aufweisen. Ein Beispiel hierfür ist der Roboter CIMON (Crew Interactive MObile CompanioN) von IBM. Dieser ist ein persönlicher Assistent für Astronauten, der zur Dokumentation der Experimente und zum Small Talk verwendet wird. Dabei stellte sich aber die Frage, wie mit den persönlichen Daten umzugehen ist. Um die Privatsphäre der Astronauten zu schützen, wurde vereinbart, die Daten aus persönlichen Gesprächen nicht weiter zu verwenden. Hier lassen sich Analogien zum Marketing bezüglich der Nutzung von Kundeninformationen ziehen.

\section{Ethische Fragen}

Da durch die KI immer mehr Daten gesammelt werden, werden Nutzer zunehmend «gläserner». Es ist deshalb nach Ansicht der Experten zwingend erforderlich, die Privatsphäre der Nutzer zu schützen und vertrauensvoll mit den Daten umzugehen. Ein weiterer Aspekt besteht darin, dass Menschen einer KI keine Fehler verzeihen: Sie sind gegenüber anderen Personen meist wesentlich nachsichtiger. Jedoch stellt sich die Frage, wer für falsche Entscheidungen verantwortlich ist: die KI oder der Mensch. Ein bekanntes Beispiel ist das Autonome Fahren. Die Debatte der Schuldfrage im Falle eines Unfalls ist nach wie vor nicht gelöst. Die Auswirkung einer Entscheidung ist entscheidend und muss deshalb differenziert werden. 


\section{Entscheidungen}

Die Experten sind sich weitgehend einig, dass die Verantwortung für falsche Entscheidungen letztlich immer beim Menschen liegt, da KI lediglich die Entscheidungsfindung unterstützt. Führungskräfte müssen somit gewährleisten können, dass sie alle relevanten Informationen in ihre Entscheidung einbezogen haben. Dies ist weitaus einfacher, wenn die KI transparenter und erklärbarer wird. Ein Beispiel ist Procter \& Gamble (P\&G). Sie nutzen KI bei der strategischen Entscheidungsfindung zur Analyse der meistverkauften Produktvarianten für jedes Geschäft und jedes Land. So können Finanz- und Verkaufsdaten kombiniert werden, um eine ganzheitliche und transparente Entscheidungsfindung zu ermöglichen.

\section{Management}

Führungskräfte sollten über ein grundsätzliches Verständnis von KI verfügen sowie offen gegenüber Innovationen sein. Dies erfordert, dass Manager ihr Entscheidungsverhalten auf einen stärker datengestützten Entscheidungsprozess anpassen. Die Deutsche Bahn nutzt KI zur Unterstützung der Verwaltung für operative Prozesse. Der Verkehr wird immer komplexer und KI erleichtert es, Probleme an den Schienen festzustellen und die weitere Vorgehensweise zu planen.

Durch mehr Wissen und Transparenz bezüglich KI wird Vertrauen in diese Technologie gestärkt, wodurch die Akzeptanzwahrscheinlichkeit steigt. IBM ermöglicht es, durch ihre KI-Anwendung Verzerrungen der Daten (biased data) zu überprüfen und eine gewisse Erklärbarkeit zu schaffen. Infolgedessen wird die Transparenz darüber erhöht, warum Entscheidungen in welcher Weise getroffen wurden.

\section{Applikationen im Marketing}

KI wird im Marketing bereits sehr häufig eingesetzt, insbesondere in der operativen Segmentierung von Kunden und im Rahmen der direkten Kundenansprache. Zumeist handelt es sich (noch) um automatisierte, operative Prozesse. Dahingegen findet KI bei strategischen Marketingentscheidungen bislang noch selten Anwendung.

Die Experten wurden im Rahmen der Studie ferner nach den grössten Schwierigkeiten, Potentialen und Herausforderungen im Umgang mit KI-Technologien gefragt, die nachfolgend kurz dargestellt werden.

\subsection{Möglichkeiten der KI im Marketing}

Die Hauptmöglichkeiten lassen sich hinsichtlich Effizienz, Produkt- und Dienstleistungsqualität, Kundenzufriedenheit und Marketingmassnabmen differenzieren.

Effizienzsteigerung durch KI

Die grösste Chance in Bezug auf den Einsatz von KI besteht in der Effizienzsteigerung. Dies wurde nahezu von allen Experten bestätigt. Der Einsatz von KI bietet Unternehmen die Möglichkeit, die Effizienz bei der Arbeit vor allem durch Automatisierung zu steigern. So können sehr präzise Vorhersagen und Prognosen in Echtzeit erstellt werden. Dies basiert auf der Fähigkeit der KI, eine sehr grosse Datenmenge zu verarbeiten und zu analysieren. Dadurch können Unternehmen effizienter in ihren Entscheidungsprozessen unterstützt werden. 
«Call Center [...], relevante Informationen bereitzustellen, egal welche Aktion sie gerade starten. [...] Aktuelle Nachrichten auswerten, Social Listening (d.h. Social Media Auswertungen), alle relevanten Informationen mit hinein zu nehmen. Und das bezieht sich meistens nicht nur auf Text, sondern man kommt sehr schnell auch in die Auswertung von Bildmaterial. Und wenn es richtig umfangreich betrieben wird, dann geht es auch in die Auswertung von Videomaterial.» (Experte 19)

Streng genommen bezieht sich KI allerdings nicht nur auf die Effizienz, sondern auch auf die Effektivität, da gewisse Datenauswertungen erst durch den Einsatz von KI möglich werden. $P \& G$ nutzt zur Entscheidungsunterstützung einen intelligenten Agenten namens SAMANTHA. Dadurch ist es erst möglich, die Menge an Verkaufsdaten zu analysieren und Prognosen über Verkaufszahlenänderungen in Echtzeit zu erstellen.

\section{Qualitätsoptimierung durch KI}

Die Qualität von Produkten und Dienstleistungen kann deutlich verbessert werden, weil Fehler minimiert werden und eine effizientere sowie präzisere Arbeitsweise sichergestellt wird. Dadurch wird auch die Beziehung zwischen dem Unternehmen und seinen Stakeholdern, wie etwa Kunden, verbessert, da ihre Qualitätsansprüche besser erfüllt werden können. Unternehmen, die in der Lage sind, Kunden und deren Verhalten zu antizipieren, können ihr Leistungsangebot und die Servicequalität gezielter optimieren.

"Derjenige, der den Kunden am besten kennt und sein Verhalten antizipieren kann, der hat gewonnen, der kann sein Unternehmen besser steuern, der kann sein Produktsortiment, seine Servicequalität anpassen und personalisierte Kundenansprache betreiben.»(Experte 5)

\section{Höhere Kundenorientierung durch KI}

Eine weitere Chance besteht in einer Verbesserung der Kundenorientierung. Da KI es ermöglicht, Kunden schneller, umfassender und personalisierter anzusprechen, können individuelle Bedürfnisse antizipiert und befriedigt werden. Unternehmen können sich kundenorientierter ausrichten und das Kundenerlebnis (Customer Experience) verbessern.

«Man versucht nicht nur unbedingt den User zu identifizieren, sondern es geht mehr darum herauszufinden, was gerade jemand liest und was ihn beschäftigt und basierend auf diesem Wissen werden Datenbotschaften ausgespielt. Das ist nur mit KI möglich.» (Experte 5)

Unternehmen wie Amazon oder Spotify setzen dabei auf Empfehlungssysteme, um die Kundenzufriedenheit zu erhöhen. Anhand vorheriger Präferenzen wird ein geeignetes Produkt vorgeschlagen. Eine höhere Kundenorientierung kann auch durch eine vorausschauende Wartung (Predictive Maintenance) erreicht werden. Dabei gibt die KI-Anwendung im Vorfeld an, wann die nächste Reparatur notwendig ist. 


\section{Anwendungen und Einsatzfelder von KI im Marketing}

Es gibt zahlreiche Anwendungsfälle, die von den Experten für das Marketing erwähnt wurden (vgl. Abbildung 5).

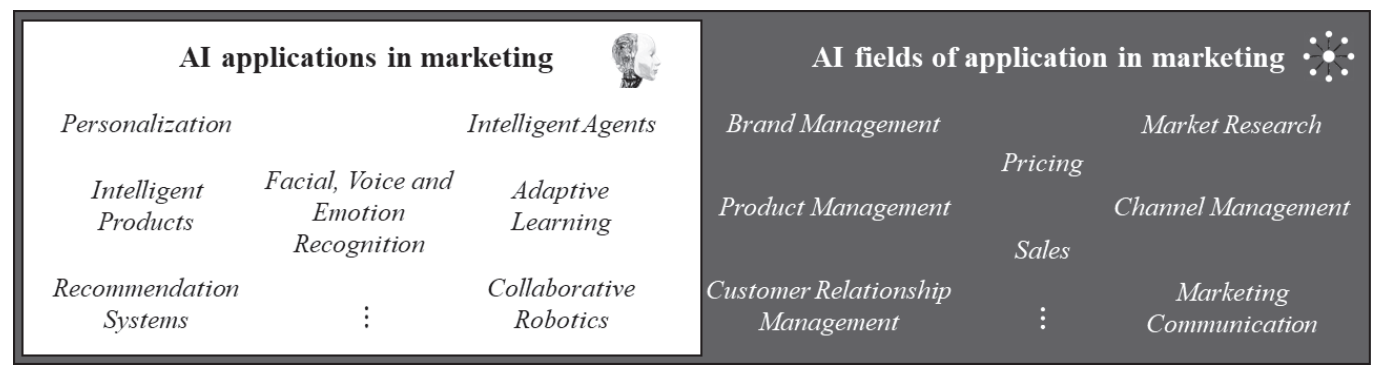

Abbildung 5: Anwendungen und Einsatzfelder von KI im Marketing ${ }^{6}$

Unter den Anwendungsgebieten im Marketing wurden drei KI-Anwendungen von den Experten hervorgehoben.

Intelligente Agenten können als Verkaufsassistenten eingesetzt werden, die dem Verkaufspersonal während des Kundengesprächs Empfehlungen und Informationen bereitstellen. Hierbei sind Chatbots eine stark verbreitete Anwendung. Sie können rund um die Uhr über die Website des Unternehmens mit dem Kunden kommunizieren. Bei der INTER Versicherung erhalten Kunden per Chatbot Antworten auf Ihre Fragen zu Versicherungspolicen.

Personalisierung wird auf Basis der KI intensiv eingesetzt. Dabei geht es um die individuelle Ansprache von Kunden. Zum Beispiel werden Kunden individualisierte Werbebotschaften auf Webseiten oder in Apps präsentiert. P\&G nutzt KI, um Kunden online sowie offline mit individuellen Nachrichten anzusprechen, indem eine Botschaft entsprechend des jeweiligen Benutzerprofils personalisiert wird.

Empfehlungssysteme werden häufig eingesetzt, um Kunden bestimmte Produkte oder Dienstleistungen vorzuschlagen, die ihren bisherigen Präferenzen entsprechen. Dies lässt sich insbesondere zur Kundenbindung im Onlinehandel einsetzen. IBM nutzt eine KI-Anwendung (Watson personality insights), um Produktempfehlungen auf der Grundlage des Persönlichkeitsprofils eines Benutzers zu geben.

Im Marketing gibt es zahlreiche weitere Einsatzfelder von KI-Applikationen:

Eine eher neue Technologie, die im Marketing eingesetzt wird, ist Computer Vision. Im Einzelhandel wird sie verwendet, um das Kundenverhalten im Geschäft zu analysieren und individuelle Botschaften auf Bildschirmen anzuzeigen, um sowohl Cross- als auch Up-Selling zu ermöglichen.

6 Die aufgelisteten KI-Anwendungen und KI-Einsatzfelder im Marketing repräsentieren einen Ausschnitt der Anwendungen, die heute verwendet werden. 
«Computer Vision wird bei der Produkterkennung, bei der Personenerkennung, bei „Mood“-Sentiment und so weiter eingesetzt. Gerade im Online-Commerce.» (Experte 7)

Darüber hinaus können mittels KI Marktanalysen schneller und effizienter durchgeführt werden. Darunter fallen zum Beispiel Kunden- und Zielgruppensegmentierung, aber auch die Erstellung von Benutzerprofilen.

"I think from a usage level, machine learning applications are the ones that are being mostly used in the context of marketing [...] One big use of it, is better understanding the markets and the consumers. So, what these models allow you to do is to have both a more sophisticated perspective about their consumer needs and how they change, but also have a more life perspective about it.» (Experte 31)

Dynamische Preis- und Marketingkampagnen können durch den Einsatz von KI optimiert werden. Videoanalysen lassen sich einsetzen, um zu analysieren, wie Kunden Produkte wahrnehmen und konsumieren. Dies wird genutzt, um Potenziale für Produktverbesserungen zu identifizieren. Ausserdem werden Kundenbeschwerdedaten mit Hilfe von Text Mining (Textanalysen) analysiert, um den gesamten Prozess zu beschleunigen.

Für die Kundeninteraktion werden vermehrt kollaborative Roboter (Collaborative Robotics) eingesetzt. Ein bekanntes Beispiel ist der Roboter Pepper von Softbank Robotics. Darüber hinaus wird die Stimmungsanalyse (Voice and Emotion Recognition) auf Grundlage natürlicher Spracherkennung auf Kundenrezensionen angewandt, um ein besseres Verständnis der Kundenhandlung zu erhalten. Ein weiteres Anwendungsgebiet im Marketing-Management ist die Produktentwicklung. Einige Unternehmen setzen KI ein, um neue Produkte zu entwickeln. Der Parfümhersteller Symrise verwendet KI als Assistenten bei der Entwicklung neuer Düfte, die auf spezielle Zielgruppen ausgerichtet sind. Dabei schnitt der von KI produzierte Duftstoff auf dem Markt am besten ab. Darüber hinaus ermöglicht Augmented Reality den Kunden, Produkte virtuell zu Hause auszuprobieren. Ein Beispiel ist das Testen von Make-up.

\subsection{Herausforderungen der KI im Marketing}

Bei den Hauptherausforderungen für die KI ist zwischen dem Einsatz und der Implementierung von KI zu unterscheiden.

\subsubsection{Implementierung von KI im Marketing}

Eine zentrale Herausforderung besteht darin, die richtigen Handlungsfelder für die jeweilige Unternehmenssituation auszuwählen und anschliessend erfolgreich zu implementieren.

\section{Fehlende Umsetzungsstrategie}

Viele Unternehmen besitzen keine klare Strategie bezüglich der Implementierung der KI. Sie wollen aufgrund des derzeitigen "Hypes" KI implementieren, ohne im Vorfeld einen expliziten Anwendungsfall identifiziert zu haben. Diesen suchen Unternehmen häufig erst 
im Anschluss nach der Einführung. Diese Strategie wird jedoch laut der Experten nicht zum Erfolg führen.

"Once you start introducing a technology, start from the simplest thing. So, the concrete example would be, don't start by saying we're going to need to build up our own neural net-based decision prediction machines from first principles. Pick something off the shelf and see how well that works for you.» (Experte 20)

Eine klare Strategie und ein fokussierter, abgegrenzter Anwendungsfall erhöhen die Erfolgswahrscheinlichkeit einer Implementierung. Es empfiehlt sich, die relevanten Mitarbeitenden in den Entscheidungsprozess bezüglich KI zu integrieren, um die Akzeptanz zu fördern.

\section{Unternehmenskultur und Managementsupport spielen eine wichtige Rolle}

Darüber hinaus kann die Unternehmenskultur für den Einsatz von KI eine Herausforderung sein. Eine risikoscheue und konservative Kultur hemmt die Implementierung. Somit sollte ein gewisses Mass an Risikobereitschaft, Agilität und Innovationsfähigkeit in der Unternehmenskultur und im Management verankert sein.

"I think the biggest difficulty today is the organizational and human factors of technology. Of course, you have the technology aspect, too. The technology works and so we are still improving the technology, which will improve over time. But then what prevents a lot of companies from leveraging this technology, it's more the fact that they're not organized for it.» (Experte 36)

\section{Ethische Herausforderungen sind komplexer als technologische Probleme}

Alle Experten sahen bezüglich des Einsatzes von KI im Marketing den Bereich der Ethik als eine äusserst schwierige und ernstzunehmende Herausforderung an. Verantwortlichkeit, Entscheidungstransparenz und Datenschutz sind diesbezüglich kritische Aspekte.

"Sie haben in komplexen Systemen, insbesondere in maschinellen Systemen, ein riesiges Komplexitätsproblem, ein riesiges Transparenzproblem und daher auch ein Erklärbarkeitsproblem. Das ist nicht mehr lösbar und dadurch, dass sie jetzt den Begriff KI für alles verwenden, überträgt das alles auf andere Bereiche.» (Experte 6)

Ein negatives Beispiel ist der Microsoft Bot Tay. Der Bot hat sich bereits nach wenigen Stunden politisch extrem rechts orientiert und musste nach nur 16 Stunden abgeschaltet werden. 


\subsubsection{Einsatz von $\mathrm{KI}$ im Marketing}

\section{Wissen über KI ist der Schlüssel}

Viele Marketingführungskräfte und -mitarbeitende verstehen nicht, wie KI-Technologien funktionieren. Sie sind daher verunsichert und skeptisch bezüglich der KI-Anwendung im Marketing. Dies wirkt sich negativ auf ihr Vertrauen in die KI aus. Viele Unternehmen sind aber auch naiv bezüglich der KI-Potenziale. Sie überschätzen das Potenzial deutlich und halten KI für eine universale Lösung, die alle Probleme auf Anhieb ohne Anstrengung lösen kann.

«Es gibt viele Unternehmen, die sagen: „, Wir wollen KI einführen, was auch immer das bedeutet.»(Experte 11)

«Die Leute glauben, sie kriegen eine Wunderkiste.» (Experte 33)

Wenn die Ergebnisse des KI-Einsatzes letztlich diese häufig unrealistischen Erwartungen nicht erfüllen, wird die KI-Technologie wieder schnell abgeschafft.

"Die Erwartungen sind einfach zu hoch und führen dann natürlich dazu, dass man sagt: so sehr hilft das nicht. [...] Man erkennt nicht, dass KI mehr als eine situative Projektrelevanz hat, sondern eine Relevanz darüber hinaus. Diese muss man mittelfristig sehen, und die Möglichkeiten und Chancen auch geistig erschließen können. Und ich glaube, da fehlt es noch ein bisschen an der Transparenz im Markt.» (Experte 34)

Daten als grösste Herausforderung beim Einsatz von KI im Marketing

Nach Meinung der Experten sind Datenzugang und -nutzung die grösste Herausforderung für Unternehmen. Dazu gehören die mangelnde Qualität der Daten, eine unzureichende Quantität der Daten und die ungenügende Datenaufbereitung und -verarbeitung. Daten sind die Quelle aller KI-Technologien: Der Output hängt daher massgeblich vom Input ab. Daten sind demzufolge laut den Experten der entscheidende Faktor für den Erfolg der Technologie.

«Die Grundlage ist bei den meisten eigentlich das Schwierigste: Dafür das Bewusstsein zu schaffen, die Daten von Anfang an richtig zu sammeln.» (Experte 8)

Eine weitere Herausforderung ist das Risiko, dass KI aufgrund verzerrter Daten scheitert. In diesem Fall wird das Modell mit falschen Daten trainiert und "lernt" falsche Dinge, was wiederum zu unzweckmässigen Empfehlungen und Entscheidungen führen kann. Zum Beispiel suchen einige Kunden online nach Produkten und vergleichen lediglich die Preise. Das ausgewählte Produkt wird aber nicht online gekauft, sondern im klassischen Einzelhandel. Dennoch erhalten sie personalisierte Onlinewerbung für das gesuchte Produkt. Diese Datenlücke wirkt sich somit auf das Marketing negativ aus. 
«Dann gibt es das Thema Bias, wie stelle ich sicher, dass ich die Modelle richtig trainiere, dass ich nicht ein Bias reinkriege.»(Experte 2)

Datensicherheit und Datenschutz sind weitere wichtige Herausforderungen. Durch die zunehmenden Möglichkeiten der Datenerhebung gewinnt der Verbraucherschutz für Unternehmen an Bedeutung. Es ist anspruchsvoll, sicherzustellen, dass keine Datenschutzprobleme auftreten und auch international alle Rechte der Verbraucher eingehalten werden.

"Ein Aspekt zum Marketing, was natürlich interessant ist, ist das Targeting von bestimmten Personen. Bei solchen Systemen sollte man immer auf die Persönlichkeitsrechte der Personen achten, die dort angesprochen werden. Es kann aber dazu führen, dass die Personen durch dieses gezielte Targeting ihrer eigenen Person abgeschreckt sind und explizit nicht bei diesem Hersteller kaufen. Das ist ein zweischneidiges Schwert.» (Experte 21)

Im Marketing gibt es durch KI ein hohes Potenzial bei der direkten Kundenansprache und der Personalisierung. Allerdings besteht die Gefahr, Kunden durch zu präzise Zielgruppenansprache abzuschrecken, indem beispielsweise Produkte vorgeschlagen werden, über die kürzlich gesprochen wurde. Aus diesem Grund ist es nicht immer ratsam, alle gesammelten Daten zu nutzen. Häufig erhöht eine vertrauliche Behandlung der Daten aufgrund des dadurch gesteigerten Kundenvertrauens den Mehrwert für den Kunden.

\section{Fazit und Ausblick}

Die Möglichkeiten und die Potenziale der KI im Marketing sind enorm. In den nächsten Jahren wird es noch deutlich mehr marketingrelevante KI-Applikationen geben. Im Rahmen dieser Trendstudie konnten praktische Erkenntnisse hinsichtlich der Möglichkeiten und Herausforderungen der KI in ihrer Anwendung im Marketing gewonnen werden. Dabei konnten schwerpunktmässig Experten aus der DACH-Region befragt werden, was eine regionale Limitierung der Aussagekraft der Studie zur Folge hat.

Aus Sicht der Experten bestehen jedoch nach wie vor zahlreiche Herausforderungen, die nicht nur Unternehmen und Führungskräfte, sondern die Gesellschaft im Allgemeinen betreffen. Nur wenn diese bewältigt werden, kann das volle Potential von KI ausgeschöpft werden. KI erfordert neue Kompetenzen, Vertrauen in die Technologie und eine grosse Veränderungsbereitschaft. Ungeachtet dieser neuen Mensch-Maschine-Interaktion bleiben noch zahlreiche Fragen offen, wie eine transparente Zusammenarbeit gewährleistet werden kann. Zudem bedürfen die ethischen Fragen einer Antwort, zum Beispiel bezüglich Verantwortung für und Erklärbarkeit von KI-basierten Entscheidungen. Aus Sicht der Forschenden sind somit weitere Forschungsanstrengungen insbesondere in den Geistesund Sozialwissenschaften erforderlich, damit KI das volle Potenzial zum Nutzen der Gesellschaft entfalten kann.

Aus diesen Ergebnissen leiten die Autoren Folgendes ab: Sollte KI ohne die richtigen Voraussetzungen eingesetzt werden, kann der Einsatz dazu führen, dass Mitarbeitende der Technologie nicht vertrauen oder sogar Angst davor entwickeln. Insbesondere wenn sie befürchten, dass ihre Arbeitskraft durch KI ersetzt werden könnte, werden sie den 
persönlichen Nutzen nicht erkennen. Dies könnte dazu führen, dass Mitarbeitende gegen die Implementierung intervenieren und somit das Unternehmen in seiner Entwicklung behindern. Werden hingegen die Mitarbeitenden in ihren Bedenken ernst genommen, ausreichend geschult und in den Implementierungsprozess integriert, kann Effektivität und Effizienz für das Unternehmen sowie der Nutzen für Kunden gesteigert werden.

\section{Literaturverzeichnis}

Bellman, A. (1978): Artificial Intelligence: Can Computers Think? Thomson Course Technology.

Charniak, E./McDermott, D. (1985): Introduction to Artificial Intelligence. Reading, MA: AddisionWesley.

Chui, M. et al. (2018): An executive's guide to AI. Verfügbar unter https://www.mckinsey.com/busin ess-functions/mckinsey-analytics/our-insights/an-executives-guide-to-ai (Zugriff am 21.08.2020).

Colson, E. (2019): What AI-Driven Decision Making Looks Like. Harvard Business Review. Verfügbar unter https://hbr.org/2019/07/what-ai-driven-decision-making-looks-like (Zugriff am 14.08.2020).

Davenport, H./Kirby, J. (2016): Just how smart are smart machines?. MIT Sloan Management Review, 57(3), S. 21.

Davenport, T. et al. (2020): How artificial intelligence will change the future of marketing. Journal of the Academy of Marketing Science, 48(1), S. 24-42.

Gentsch, P. (2019): Künstliche Intelligenz für Sales, Marketing und Service. New York, NY: Springer Publishing.

Gioia, A. et al. (2013): Seeking qualitative rigor in inductive research: Notes on the Gioia methodology. Organizational research methods, 16(1), S. 15-31.

Glaser, B./Strauss, L. (1967): The Discovery of Grounded Theory. Chicago: Aldine.

Huang, H./Rust, T. (2018): Artificial intelligence in service. Journal of Service Research, 21(2), S. 155-172.

Jaeger, U./Reinecke, S. (2009): Expertengespräch. In: Baumgarth, C. et al. (Hrsg.): Empirische Mastertechniken: eine anwendungsorientierte Einführung für die Marketing- und Managementforschung. Wiesbaden: Gabler, S. 29-76.

Kaplan, A./Haenlein, M. (2019): Siri, Siri, in my hand: Who's the fairest in the land? On the interpretations, illustrations, and implications of artificial intelligence. Business Horizons, 62(1), S. $15-25$.

Kietzmann, J. et al. (2018): Artificial intelligence in advertising: How marketers can leverage artificial intelligence along the consumer journey. Journal of Advertising Research, 58(3), S. 263-267.

Kolbjørnsrud, V. et al. (2016): How artificial intelligence will redefine management. Harvard Business Review, 2.

Kurzweil, R. (1990): The age of intelligent machines. MIT Press Cambridge, MA, USA.

Liebold, R./Trinczek, R. (2009): Experteninterview. In: Kühl, S. et al. (Hrsg.): Handbuch der Organisationsforschung. Quantitative und Qualitative Methoden. Wiesbaden, S. 32-56.

McCarthy, J. et al. (1955): A proposal for the Dartmouth Summer Research Project on Artificial Intelligence. Research Project on Artificial Intelligence. Verfügbar unter http://www-formal.stanf ord.edu/jmc/history/dartmouth/dartmouth.html (Zugriff am 20.07.2020).

McCarthy, J. (2007): What is artificial intelligence? Basic Questions. Computer Science Department, Stanford University. Verfügbar unter https://stanford. io/21So373 (Zugriff am 20.07.2020). 
Morse, M., et al. (2002): Verification strategies for establishing reliability and validity in qualitative research. International journal of qualitative methods, 1(2), S. 13-22.

Nilsson, J. (1998): Artificial Intelligence: A New Synthesis. Morgan Kaufmann Publishers.

Palinkas, A. et al. (2015): Purposeful sampling for qualitative data collection and analysis in mixed method implementation research. Administration and policy in mental health and mental health services research, 42(5), S. 533-544.

Rich, E./Knight, K. (1991): Artificial Intelligence. Artificial Intelligence Series. 2. Auflage. McGrawHill.

Russell, S./Norvig, P. (2010): Artificial intelligence: A modern approach. Upper Saddle River: Prentice-Hall.

Rust, T. (2020): The future of marketing. International Journal of Research in Marketing, 37(1), S. 15-26.

Torra, V. et al. (2019): Artificial intelligence, In: Said, A./Torra, V. (Hrsg.): Data science in practice. Springer, S. 9-26.

Traumer, F. et al. (2017): Towards a Future Reallocation of Work between Humans and Machines - Taxonomy of Tasks and Interaction Types in the Context of Machine Learning. International Conference on Information Systems (ICIS). Seoul, South Korea.

Wassermann, S. (2015): Das qualitative Experteninterview. In: Niederberger, M./Wassermann, S. (Hrsg.): Methoden der Experten-und Stakeholdereinbindung in der sozialwissenschaftlichen Forschung, Springer VS, Wiesbaden, S. 51-67.

Wilson, J./Daugherty, R. (2018): Collaborative intelligence: humans and AI are joining forces. Harvard Business Review, 96(4), S. 114-123.

Wolan, M. (2020): Künstliche Intelligenz verändert alles. In: Wolan, M. (Hrsg.): Next Generation Digital Transformation. Springer Gabler, Wiesbaden, S. 25-50.

Gioia Volkmar, M.Sc., ist wissenschaftliche Mitarbeiterin am Institut für Marketing \& Customer Insight an der Universität St. Gallen (HSG).

Anschrift: Institut für Marketing \& Customer Insight, Universität St. Gallen, Dufourstrasse 40a, 9000 St. Gallen, Schweiz, Tel.: +41 (0) 71/224-2831, E-Mail: gioia.volkmar@unisg.ch

Sven Reinecke, Dr., ist geschäftsführender Direktor des Instituts für Marketing \& Customer Insight sowie Titularprofessor an der Universität St. Gallen (HSG).

Anschrift: Institut für Marketing \& Customer Insight, Universität St. Gallen, Dufourstrasse 40a, 9000 St. Gallen, Schweiz, Tel.: +41 (0) 71/224-2873, E-Mail: sven.reinecke@unisg.ch

Peter Mathias Fischer, Dr., ist Assistenzprofessor am Institut für Marketing \& Customer Insight an der Universität St. Gallen (HSG).

Anschrift: Institut für Marketing \& Customer Insight, Universität St. Gallen, Dufourstrasse 40a, 9000 St. Gallen, Schweiz, Tel.: +41 (0) 71/224-2888,

E-Mail: peter.fischer@unisg.ch 